\title{
Nanofibrous piezoelectric structures for composite materials to be used in electrical and electronic components
}

\author{
D. Fabiani ${ }^{1}$, F. Grolli ${ }^{1}$, G. Selleri ${ }^{1}$, M. Speranza ${ }^{1}$, T. M. Brugo ${ }^{2}$, E. Maccaferri ${ }^{2}$, D. Cocchi ${ }^{2}$ \& A. Zucchelli ${ }^{2}$ \\ ${ }^{1}$ DEI - University of Bologna, Bologna, Italy \\ ${ }^{2}$ DIN- University of Bologna, Bologna, Italy \\ davide.fabiani@unibo.it
}

\begin{abstract}
This paper deals with realization of multifunctional composite materials, having piezoelectric effect. First of all polymeric mats of electrospun piezoelectric nanofibers were realized with different geometries. Such effect has been maximized by designing properly the electrospinning apparatus in order to enhance the electric field in the interelectrodic space which polarize the dipolar moments. The mats are then integrated in a silicon rubber matrix and measurements of the electromechanical response of the composite materials thus manufactured are performed. A good integration of nanofibers inside the host material is evidenced by electron microscopy images, allowing delaminations, which could occur using piezoelectric films, to be avoided. A large electrical response to both impact and vibration stimuli has been finally demonstrated.
\end{abstract}

\section{Introduction}

Piezoelectric materials are frequently used for energy harvesting applications and for the realization of electronic sensors for mechanical vibration analysis or structural monitoring. Among these, polymeric piezoelectrics are often preferable to ceramic materials for many applications thanks to the possibility of realizing electroactive devices in the shape of thin films, even if their piezoelectric response is lower compared to that of ceramics. Poly(vinylidene fluoride) (PVdF) and its copolymers are considered the most promising candidates, due to the strong electrical dipole moment of the PVdF monomer unit, which is related to the electronegativity of fluorine atoms compared to those of hydrogen and carbon atoms. Furthermore, PVdF shows piezoelectric behavior only in a peculiar polar phase, i.e. $\beta$-phase, due to the non-centrosymmetric conformation of the crystal [1]. Generally, mechanical stretching of the non-polar phase $(\alpha)$ can induce a partial transformation into the polar $\beta$-phase [2]. The use of PVdF co-polymers, such as PVdF-TrFE, induces $\beta$-phase formation [1] and the ferroelectric domains of $\beta$-phase must be aligned in order to provide piezoelectric behavior, e.g. by the application of an electric field.

However, if these polymeric films have to be used in a composite system constituted of a stack of different materials or components, structural problems may appear. Indeed, if installed inside other materials a polymer film could incept delaminations with the host material during mechanical stress cycling, leading to cracking formation and thus mechanical failure. On the contrary, if these polymeric piezo devices are realized in the form of nanofibrous mat, having porosity of about $70-80 \%$, the host material can penetrate between the fibers creating thus an intimate contact, which avoids delaminations to occur.

Electrospinning is claimed to be a versatile technique to manufacture nanofibrous and porous polymeric materials, e.g. PVdF-based polymers. These nanostructures are characterized by having high surface to volume ratio and a huge porosity [3, 4]. Literature papers indicate that most of reported waveforms generated by electrospun PVdF-based polymers, when subjected to mechanical stress, are associated to the piezoelectric effect [5, $6]$. This is generally associated to the presence of polar $\beta$-phase in the material. However, $\beta$-phase must be oriented through electrical poling in order to obtain a piezoelectric behavior. It is stated that electrospinning provides both the stretching of the polymer and the electrical poling of polymer dipoles, due to the application of a strong electric field during material manufacturing [7]. In particular, the stretching of the electrostatically charged jet induces a partial transformation from $\alpha$ to $\beta$ crystalline phase, which can be oriented during fiber formation. On the other hand, conventional PVdF-based thin films, usually realized by solvent casting or spin coating, require post-processes in order to exhibit the piezoelectric behavior, i.e. mechanical stretching and electrical poling. However if the electric field used in the electrospinning process is not high enough, dipole polarization could be partial or even absent, therefore the resulting electromechanical response of the material could be associated more with electrostatic effects, like e.g electric charges trapped during electrospinning process, than due to piezoelectric behavior [8]. Trapped charged, however, are not stored in PVdF for long time, due to its relatively high conductivity, therefore this effect decreases with storage time and disappears after about 200 days.

In this work, a deep study of the electric field used in the electrospinning process is carried out in order to obtain polarized piezoelectric nanofibers having different geometries which can then be integrated in a polymeric matrix. Measurements of the electromechanical response of the composite materials thus manufactured is finally carried out and comparison between the different geometries is performed. 


\section{Materials and Methods}

Two fiber geometries were investigated in this paper: stiff cylindric nanofibers and core-shell coaxial nanofibers. The formers are entirely realized of piezoelectric polymer while the latters have a conductive core and a piezoelectric shell, thus realizing a coaxial nano-piezoelectric device (see Fig. 1).

PVdF-TrFE Solvene $\left(75 / 25 \mathrm{~mol} \%, \mathrm{M}_{\mathrm{w}}=410 \mathrm{kDa}\right)$ kindly provided by Solvay Specialty Polymers (Bollate, Italy), was used as piezoelectric material. For the stiff fibers, the polymer solution was prepared dissolving the polymer at a concentration of $15 \% \mathrm{w} / \mathrm{v}$ in Acetone: Dimethyl-formamide (DMF) (70:30 $\mathrm{v} / \mathrm{v})$ and further electrospun using the electrospinning conditions optimized after numerical simulation reported in the following section. For the core-shell fibers two solutions were prepared. Shell solution was realized dissolving $12 \% \mathrm{w} / \mathrm{v}$ of the same PVdF-TrFE in MEK:DMF 75:25. The main problem for this fiber geometry is related to optimization of the core solution. In order to obtain fiber core which could be used as electrode, a conducting polymer, namely PEDOT:PSS, is used. Since this material cannot be electrospun directly, a carrying polymer has to be used, e.g. polyvinylpyrrolidone (PVP). Therefore a solution of PEDOT:PSS and PVP $82 \%-18 \%$ in DMF has been prepared. The exact amount of DMF was varied and optimized to maximize viscosity of core solution with respect to shell solution so that the two coaxial jets form two separate phases during the electrospinning process. A concentration of about $67 \% \mathrm{w} / \mathrm{v}$ of polymer was found to be the optimal amount.

The electrospinning apparatus used to manufacture the fibrous mat was a Spinbow Lab Unit (Spinbow S.r.l., Italy) equipped with normal or coaxial needles for the production of stiff and core-shell nanofibers, respectively, see Fig. 2. Nanofibers are produced by applying a high-field to a polymer solution. The electrostatic forces create a whirling region where the polymer jet is stretched while the solvent evaporates [3]. This results in the formation of a fiber having the diameter $<1 \mu \mathrm{m}$. Then the fiber is collected on a grounded plate, forming a nonwoven polymer mat. Depending on the kind of collector used, fibers having different alignment can be obtained, e.g. random or aligned fibers.

Membranes of $10 \times 10 \mathrm{~cm}^{2}$ and about $80-100 \mu \mathrm{m}$ thickness were manufactured. Fiber morphology was observed by means of a scanning electron microscope (PHENOM PROX Desktop SEM). Fiber diameter distribution was evaluated through PHENOM Fibermetric software. Electrospun samples $\left(3 \times 4 \mathrm{~cm}^{2}\right)$ were then immersed in liquid polydimethylsiloxane (PDMS) which was uniformly applied on the sample by using a spincoater. Finally the composite PVdF nanofibers / PDMS was cured at $90^{\circ} \mathrm{C}$ for 45 minutes. The final material is completely transparent, since nanofibers, thanks to their dimensions ranging between $200 \mathrm{~nm}$ and $400 \mathrm{~nm}$, do not reflect visible light.

PDMS nanocomposite sample are finally metallized on both sides and sandwiched between aluminum-foil electrodes. An electrical piston was used to test the electrical response of the samples, when subjected to a mechanical impulse generated by the piston mass of about $100 \mathrm{~g}$ falling on the sample from $3 \mathrm{~cm}$ heigth at a given repetition rate

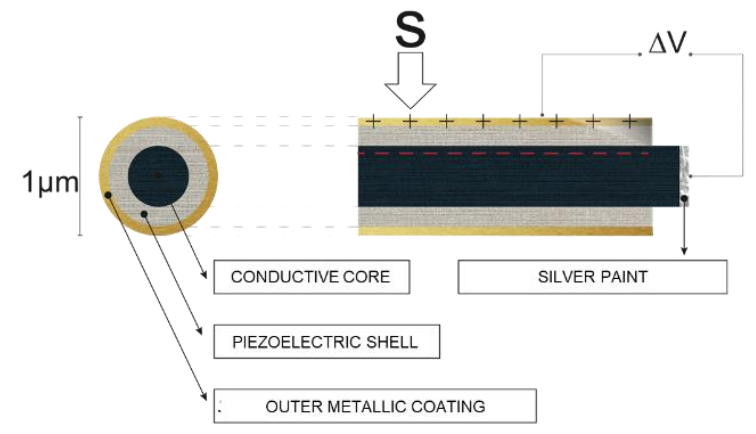

Figure 1. Sketch of core-shell fibers.

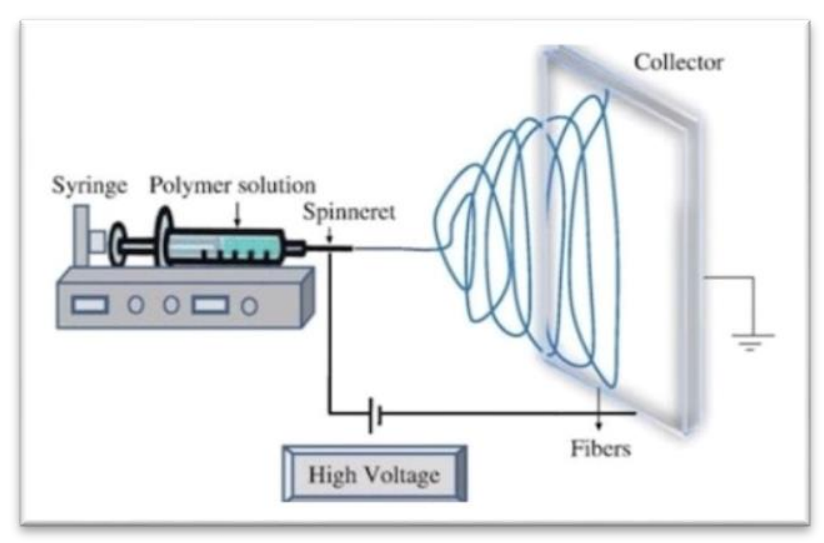

Figure 2. Scheme of an electrospinning unit.

\section{FEM Simulations}

Finite Element Modelling (FEM) simulations were carried out using COMSOL with the aim of investigating the behavior of the electric field in the air gap between the injecting needle and the counter electrode where the electrospun fibers are collected. This analysis is important because we need to orient the dipole moments of the piezoelectric fibers when they travel from the needle to the collecting electrode, therefore higher field in the gap will provide larger polarization [9].

A sketch of the simulated geometry is reported in Fig. 3. The electric field behavior is plotted in Fig. 4 for different distances between needle and collector, precisely $150 \mathrm{~mm}$ (Fig. 4A), 90 $\mathrm{mm}$ (Fig.4B) and $60 \mathrm{~mm}$ (Fig. 4C). Voltage applied to the needle (positive) and to collector (grounded or negative) is also varied.

Simulations show that under this highly divergent field configuration, typical distance needle/collector of $150 \mathrm{~mm}$ produce quite low field in the interelectrodic space even at high applied voltages, while reducing the electrode distance and increasing the voltage difference, the field in the middle can increase significantly. The best conditions are reached with electrode distance of $60 \mathrm{~mm},+25 \mathrm{kV}$ and $-6 \mathrm{kV}$ applied to needle and collector, respectively. Note that a voltage $>25 \mathrm{kV}$ cannot be applied to the needle otherwise corona discharges can perturbate the electrospinning jet. It is worth noting that the collector is usually grounded in most of the electrospinning apparatus. However, a negative voltage applied, provides beneficial effects in terms of air-gap field increase allowing a stable electrospinning process up to $-6 \mathrm{kV}$. 


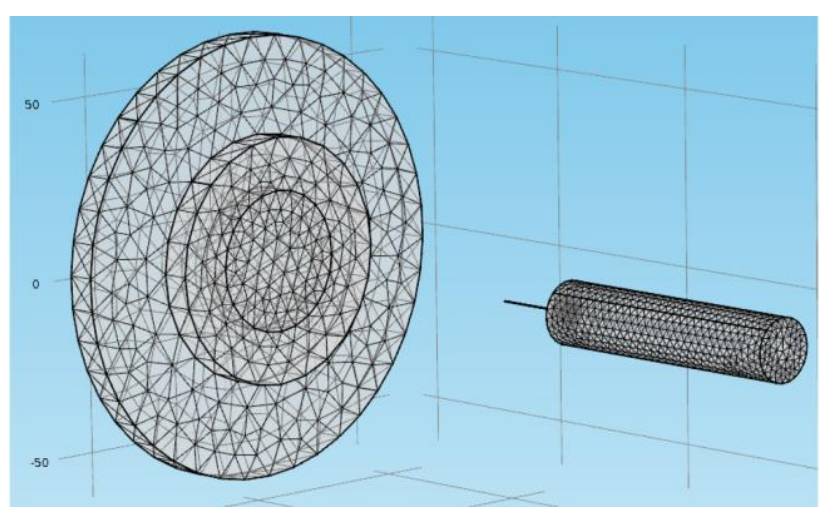

Figure 3. Sketch of HV needle-collector geometry of electrospinning apparatus with the mesh used for FEM calculations.
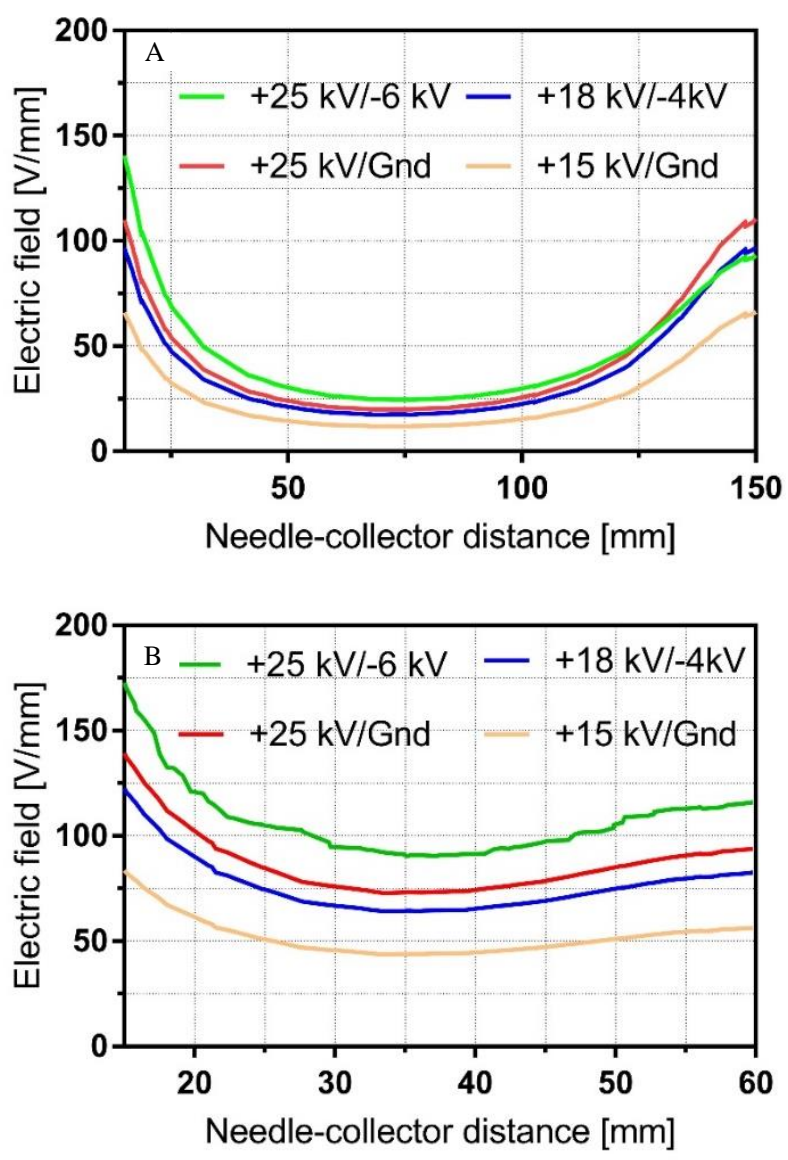

Figure 4. FEM results. Interelectrodic electric field (max value) as a function of the position from the needle for different voltage applied to the needle $(+)$ and the collector (- or ground). Needle-collector distance of $150 \mathrm{~mm}(\mathrm{~A})$ and $60 \mathrm{~mm}(\mathrm{~B})$.

\section{Experimental Results}

SEM analysis of stiff nanofibrous sample shows good quality nanofibers of PVdF-TrFE (average fiber diameter about $350 \pm 100 \mathrm{~nm}$ ) without any bead-like defect, as can be evidenced in Figure 5.

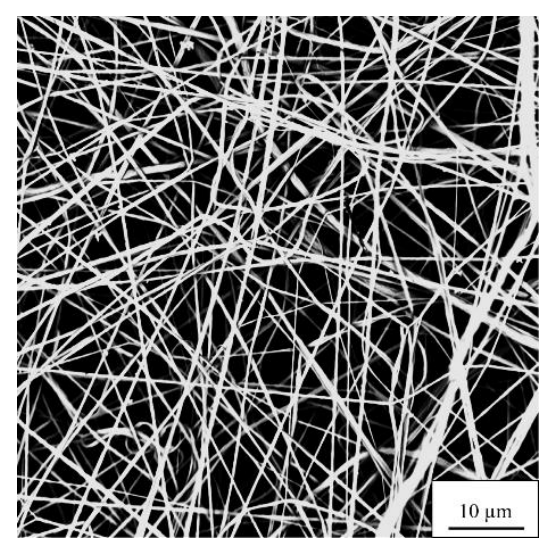

Figure 5. SEM picture of electrospun PVdF-TrFE nanofibers.

Morphology of core-shell nanofibrous samples can be observed by looking at SEM images of the cross-section obtained through fragile fracture of the sample in liquid nitrogen. The sample is then immersed in water for $2 \mathrm{~h}$ to remove PEDOT:PSS-PVP from the fiber core and finally dried in vacuum over for $6 \mathrm{~h}$. Figure 6 shows nice pictures at $5000 \mathrm{X}$ (Fig. 6A) and 10000X (Fig. 6B) magnification of a sample crosssection. A hollow structure of the fibers can be clearly evidenced, confirming that the electrospun fibrous material realized has a core-shell structure.
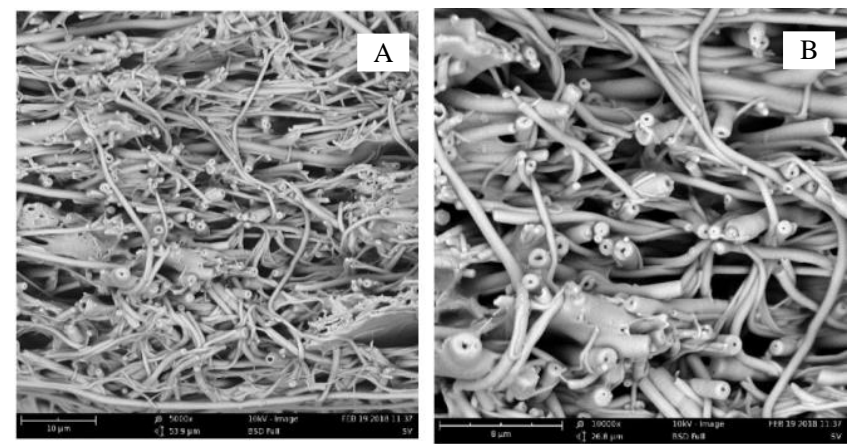

Figure 6. SEM pictures of cross-section of core-shell nanofibers. Magnifications: 5000X (A), 10000X (B)

A microscopy of PDMS sample cross-section containing the nanofibrous mat is shown in Fig. 7 at $2500 \mathrm{X}$ (Fig. 7A) and 7000X (Fig. 7B) magnifications. The pictures show a very good penetration of PDMS inside the fiber mat, thus confirming the excellent compatibility of PDMS and PVdF-TrFE nanofibers, which will avoid delaminations when periodic mechanical stresses are applied.

The electromechanical response of the realized composite material is tested by means of mechanical impacts. A very large peak-to-peak signal of about $10 \mathrm{~V}$ can be observed at each impact with the specimen, see Fig. 8A. The waveform shows the typical shape generated by a piezoelectric material, composed of two contributions of opposite polarity, one associated to material deformation (press) and one to material relaxation (release), see Fig. 8A. Moreover, reversing the electrospun sample and keeping the same voltmetric connection, signal polarity changes accordingly, see Fig. 8B, further confirming the piezoelectric nature of the realized composite material. An impact test on a blank sample, i.e. made of PDMS without any nanofiber inside 
shows a negligible electrical response to mechanical impact (see Fig. 9). The signal of $300 \mathrm{mV}$ amplitude could be associated to the triboelectric effect between PDMS specimen and the electrode, due to their relative motion.

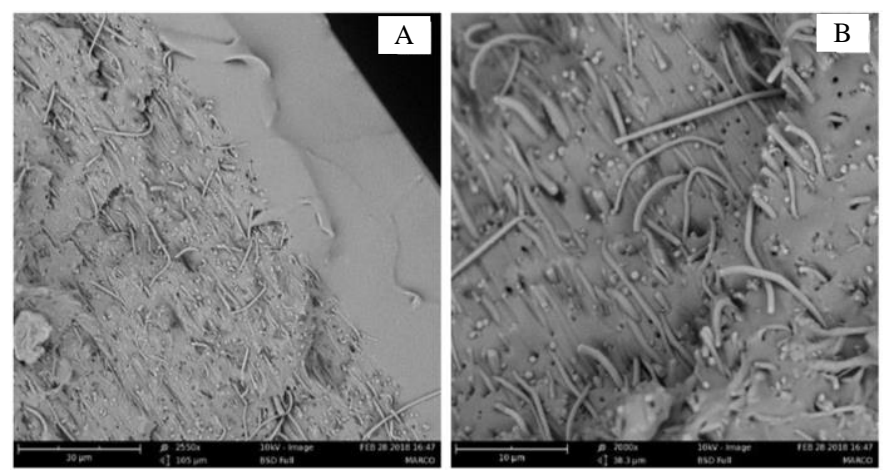

Figure 7. SEM pictures of PDMS + nanofibrous mat sample. Magnifications: 2500X (A), 7000X (B)
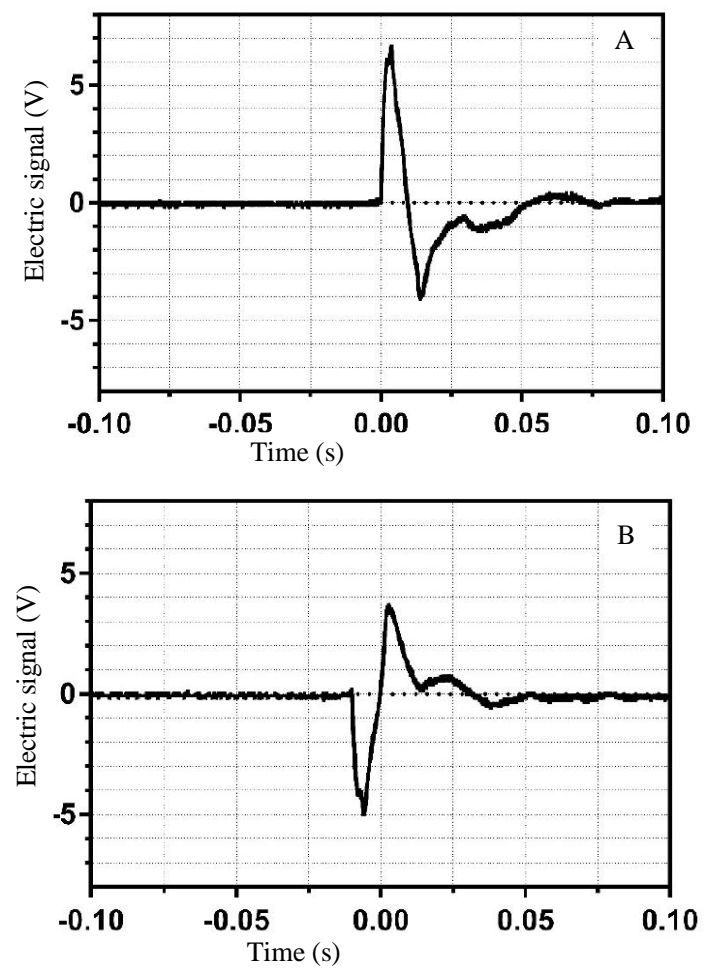

Figure 8. Electric response to mechanical impact of $100 \mathrm{~g}$ mass falling from $3 \mathrm{~cm}$ height. Sample in direct (A) and inverted (B) position.

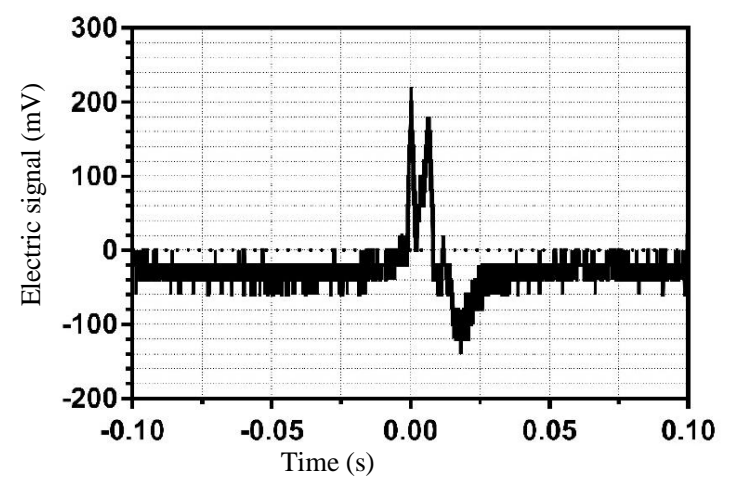

Figure 9. Electric response to the same mechanical impact of Fig. 6 for pure PDMS sample.
An example of electric signals obtained from two different electric field configuration is reported in Fig. 10. The traditional electrospinning process (needle at $15 \mathrm{kV}, 15 \mathrm{~cm}$ distant from the grounded) provides peak-to-peak signals that are 3 times smaller than those obtained in the optimized field conditions, obtained in the previous section by simulation.

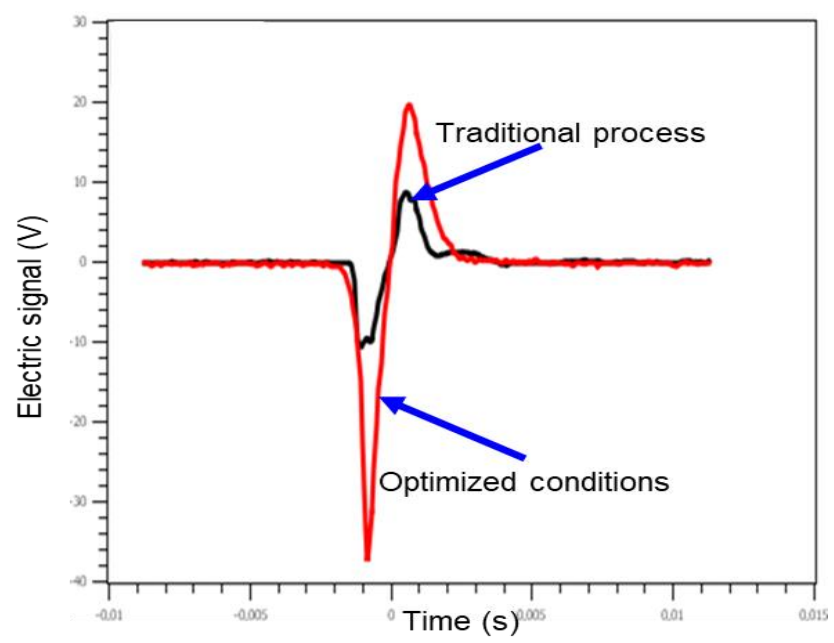

Figure 10. Electromechanical response of piezoelectric nanofibers obtained through a traditional and optimized electrospinning process.

Finally, the electrical response of the composite material has been tested in a vibrating-bench setup with acceleration ranging between 0 and $20 \mathrm{~m} / \mathrm{s}^{2}$, see Fig. 11 . It is noteworthy that the amplitude of the electric signal generated has an almost linear behavior with the acceleration applied to the specimen, which makes it suitable for applications as acceleration/vibration sensor.

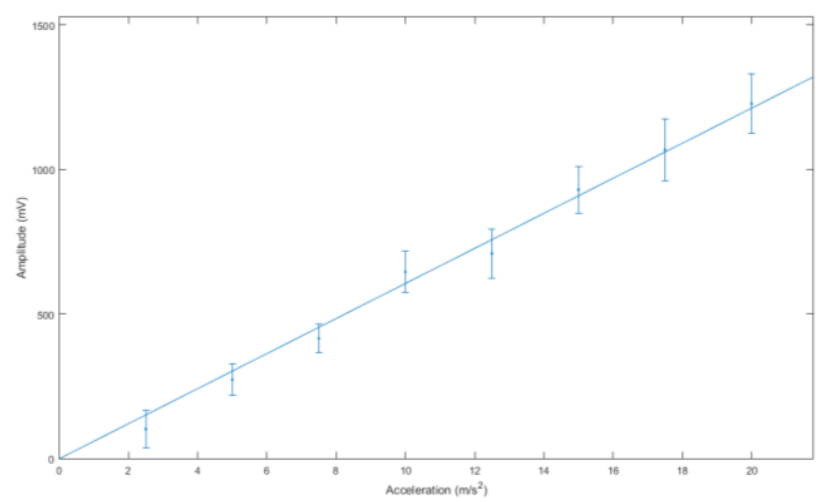

Figure 11. Electric response as a function of acceleration under vibration tests.

Despite the theoretical possibility of obtaining nanometric piezoelectric sensor from each core-shell nanofiber for practical reasons the sensors were realized by bundling some fibers together (see Fig. 12). This allow the electric signal to be better collected from the cores connected in parallel and the outer screen. In this particular configuration fiber alignment plays a major role in enhancing the electromechanical response. Indeed, signals obtained from sensors with the $80 \%$ of aligned fibers show amplitudes about 4 times larger than those having only the $30 \%$ (Fig. 13). This is explained considering that the parallel 
connection reported in Fig. 12 is more effective with aligned than random fibers.

A possible application of such nanometric sensors is reported in Fig. 14 where a $5 \times 5$ matrix constituted by bundles of single core-shell nanofibers is sketched. With such configuration a very fine position/pressure sensor, having submicrometric scale, can be realized and integrated in different hosting/embedding devices. Analyzing the signal connected from the terminals AVx, a precise location of the pressure position can be obtained quite effectively.

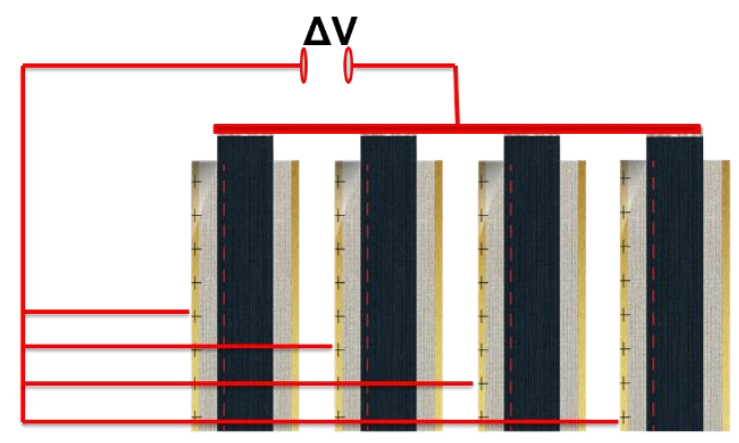

Figure 12.Parallel connection of some core-shell nanofibers to improve signal

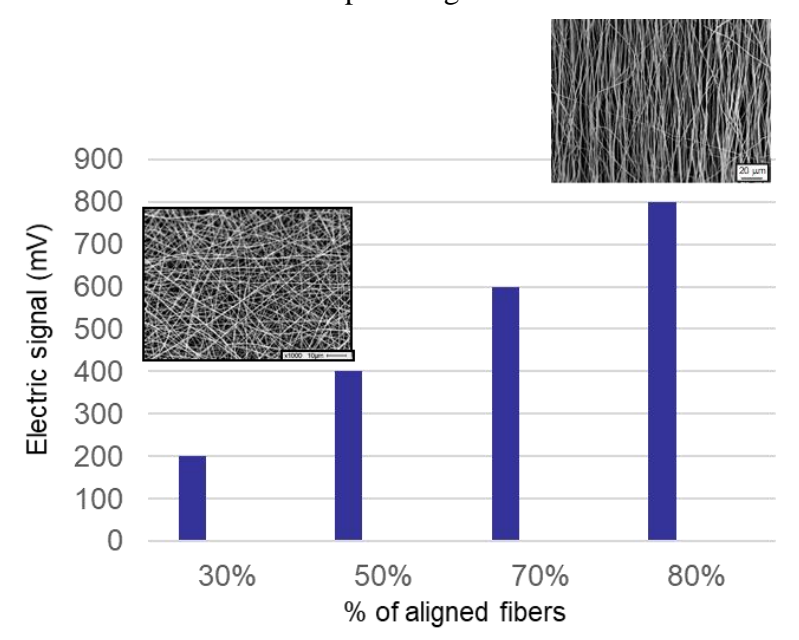

Figure 13. Electric response as a function of acceleration under vibration tests.

\section{Conclusions}

PVdF-TrFE nanofiber mats have been electrospun with different geometries and integrated inside silicone rubber, allowing this latter to exhibit a piezoelectric response when mechanical stresses are applied to the specimen, thus opening up the feasibility of realizing multifunctional smart materials. The integration of piezoelectric nanofibers is possible also in other hosting materials, like e.g. epoxy resins or different composite materials. This means that impact and/or acceleration sensors could be integrated directly in the component material, without risk of delamination thanks to the intimate contact between nanofibers and hosting materials. The use of a piezoelectric film, on the contrary, would lead to possibile delamination under periodic mechanical stresses. Applications of these multifunctional materials could be envisaged, e.g, in automotive and aircraft industry, as impact / vibration sensors and vibrational energy harvesters for low-power electronic components.

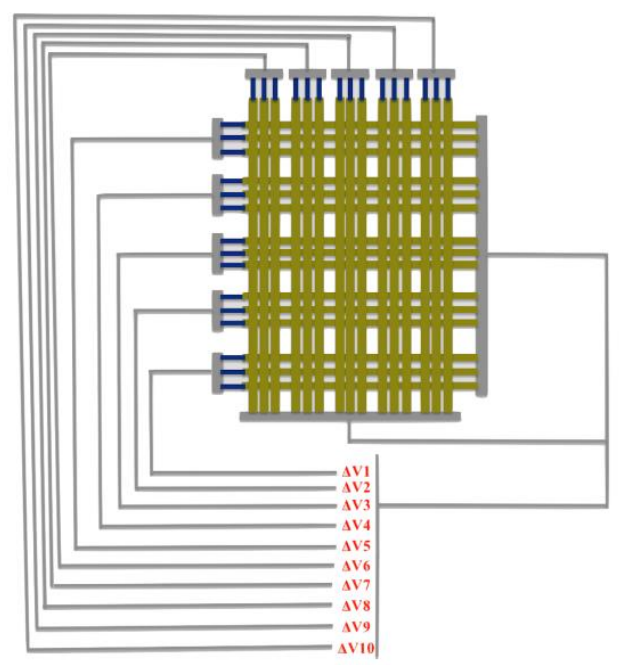

Figure 14. Electric response as a function of acceleration under vibration tests.

\section{References}

[1] P. Martins, A.C. Lopes, S. Lanceros-Mendez, "Electroactive phases of poly(vinylidene fluoride): Determination, processing and applications", Progress in Polymer Science, Vol. 39, 683-706, 2014.

[2] J. Gomes, J. Serrado Nunes, V. Sencadas and S. Lanceros-Mendez, "Influence of the $\beta$-phase content and degree of crystallinity on the piezoand ferroelectric properties of poly(vinylidene fluoride)", Smart Mater. Struct., Vol. 19, 6, 065010, 2010.

[3] S. Ramakrishna, K. Fujihara, W. E. Teo, T. C. Lim, Z. Ma (Eds), “An Introduction to Electrospinning and Nanofibers", World Scientific Publishing Co. Pte. Ltd, NJ, USA 2005.

[4] C. Burger, B. S. Hsiao, and B. Chu, "Nanofibrous materials and their applications", Annu. Rev. Mater. Res. 2006. 36:333-68.

[5] M. Baniasadi, Z. Xu, S. Hong, M. Naraghi, M. Minary-Jolandan, "Thermo-electromechanical Behavior of Piezoelectric Nanofibers", ACS Applied Materials and Interfaces, Vol. 8, 2540-2551, 2016.

[6] G. Ico, A. Showalter, W. Bosze, S. C. Gott, B. S. Kim, M. P. Rao, N. V. Myung, J. Nam, "Size-dependent piezoelectric and mechanical properties of electrospun P(VDF-TrFE) nanofibers for enhanced anergy harvesting", J. Mater Chem A, Vol. 4, 2293-2304, 2016.

[7] J. Pu, X. Yan, Y. Jiang, C. Chang, L. Lin, "Piezoelectric actuation of direct-write electrospun fibers", Sensors and Actuators A: Physical Vol. 164, Issues 1-2, 131-136, 2010.

[8] M. Zaccaria, D. Fabiani, A. Zucchelli, J. Belcari, O. Bocchi, T. Cramer and B. Fraboni, "Electret behavior of electrospun PVdF-based polymers" Proc. of IEEE CEIDP 2016, pp. 137-140, 2016.

[9] D. Fabiani, F. Grolli, M. Speranza, S. V. Suraci, T. M. Brugo, A. Zucchelli, E. Maccaferri, "Piezoelectric nanofibers for integration in multifunctional materials", Proc. of IEEE CEIDP 2018, pp. 14-17, 2018.

\section{Acknowledgemnts}

The authors are grateful to A. Marrani of Solvay Specialty Polymers (Bollate, Italy) for providing PVdF-TrFE raw material. 